\title{
Discrete particle simulation of gas-solid flows $\dagger$ (From dilute to dense flows)
}

\author{
Yutaka Tsuji \\ Department of Mechanical Engineering, \\ Faculty of Engineering \\ Osaka University*
}

\begin{abstract}
Increasing computer power has made discrete particle simulation a practical means for predicting particle motion in industrial processes. Simulation techniques for various particle motions, from dilute to dense phase flows, are explained here. In simulations of this kind, the most important problem is how to model the interactions of particles with walls, with other particles and with the fluid. Calculations based on various models for these interactions are discussed. Particle segregation phenomena, particle mixing in a rotating vessel, dense phase pneumatic conveying, and fluidized bed are shown as examples of simulations which have been made in the author's laboratory.
\end{abstract}

\section{Introduction}

Since numerical simulation of single phase flows is considered to have attained maturity, attention is being paid to multiphase flows. Fields in which such numerical simulations will play an important role as a prediction method are widespread. For example, flows in manufacturing industries, flows in nature and flows in living bodies are often multiphase. In the simulation of multiphase flows, we must treat both the fluid phase and discrete phase motions. Owing to the great achievements in single phase flows, computation techniques for fluid motion have been established satisfactorily. Now techniques for particle motion are being developed and most techniques can be applied to powder technology.

Multiphase flows are classified into the following four categories; gas-liquid, liquid-solid, gas-solid and gas-liquid-solid. As far as formulation is concerned, the gas-solid flow is among the simplest, because mass transfer and surface phenomena which make the formulation complicated need not usually be taken into account. Simulation of gas-solid flows started from dilute phase flows where particles are dispersed in gas at a low concentration. In the recent several years, the author's research group in Osaka University has been interested in flows with high concentrations. The higher the particle concentration, the stronger the impact of simulation on practical applications. For instance, dense phase flows are closely

\footnotetext{
* Suita, Osaka 565 Japan

$\dagger$ Received July 8, 1993
}

related to powder technology. Before, it seemed a dream for engineers working in powder technology to design machines based on computer simulation, but the situation is gradually changing. Nowadays computers enable us to deal with the motion of individual particles in dense phase flows where particles are in contact with each other.

\section{Eulerian vs. Lagrangian}

According to the wellknown classification, numerical methods of multiphase flows are classified as Eulerian or Lagrangian depending on the treatment of the particulate phase. The Eulerian method regards the particulate phase as a continuum. This method is further divided into one-fluid (homogeneous) and two-fluid models. An advantage of the Eulerian method is to apply techniques developed for single phase flows to the particulate phase with fewer modifications. However, it should be noted that a sufficient number of particles must be included uniformly in a control volume for calculation in order to use the Eulerian method. Unfortunately, cases satisfying this condition are limited.

The Lagrangian method computes the motion of individual particles. Its basic ideas and formulations are simple compared with the Eulerian method. However, as the number of particles increases, large memories and long computation time are needed. If the Lagrangian calculation is to be performed rigorously for all the particles in a practical multi-phase 
flow, enormous amount of memory and computation time would be required. This is a substantial disadvantage of the Lagrangian method. Fortunately, progress in computer hardware development is still continuing, which is helpful to the Lagrangian method. Also, our group has made an attempt to apply the DSMC (Direct Simulation Monte Carlo) method, developed in molecular dynamics, to multi-phase flows to avoid the above demerit of the Lagrangian method.

The above classification has been applied to dispersed phase flows in general. The same classification holds true for dense phase flows or granulate flows where the particle concentration is so dense that the particles are in continuous contact with each other. When the Eulerian method is used for such dense phase flows, the most important assumption which has to be made is about the relation between stress and strain of the continuum consisting of particles. Many efforts have been made to obtain this relation, experimentally and theoretically. However, it is not easy to deduce relations for general use.

It has not been long since the Lagrangian method was applied to dense phase flows. Compared with the Eulerian method, the Lagrangian method requires less assumptions and is expected to be a powerful means for predicting phenomena in powder technology.

\section{Collision}

\subsection{Irregular bouncing}

In dilute phase pneumatic conveying, the gas flow gives momentum and kinetic energy to the particles. That is, particles are accelerated by gas through the drag force. If a particle in a horizontal pipe did not collide with the wall, the particle velocity would be equal to the gas velocity. However the time-averaged particle velocity is always smaller than the gas velocity, because the particles lose momentum due to collisions with the wall. Since the additional pressure drop due to the particles corresponds to the fluid drag which is related to the velocity difference between the fluid and the particles, the energy loss caused by collision must be estimated precisely in the simulation.

Although particle-to-wall collision is an important phenomenon in gas-solid pipe flows, the Eulerian method has disadvantages concerning collision because the effects of collision can not be considered in a direct way. In contrast, the Lagrangian method is suitable for dealing with the collision problem. When the coefficients of restitution and dynamic friction are given as particle physical properties, the collision problem can be solved using the classical dynamics of a rigid body. However, the problem is not as simple as expected. That is, if the simulation were based on the simple assumption that spherical particles collide with a flat plate with a coefficient of restitution smaller than one, particles colliding repeatedly with the wall in a long horizontal pipe would ultimately slide on the wall. To avoid such an unrealistic result, we must take the irregularity of collisions into consideration. If the irregularity is neglected, particles can not be suspended in the gas. Previously, Magnus or shear lift forces and fluid turbulence were often considered as mechanisms suspending particles in a horizontal pipe. It is true that these fluid dynamic forces act on particles and that the effect of these forces are dominant for small particles, but the mechanism suspending large particles in horizontal pipes is the irregular bouncing of the particles against the wall. The irregularity is caused by particle shape deviating from a true sphere and roughness of the wall.

Regarding the irregular bouncing, several models have been proposed so far (Matsumoto et al. 1970a, 1970b, Tsuji et al. 1987, Tsuji et al. 1989, Frank et al. 1991). In general the relation between particle velocities before and after collision are derived using the impulsive equations with the coefficient of restitution and friction coefficient given. We (Tsuji et al., 1991) attributed the irregularity to the non-sphericity of particle shape, and demonstrated a calculation method for three dimensional collision of a nonspherical particle. This method is applicable to particles of arbitrary shape.

We calculated the trajectories of nearly spherical particles in a two-dimensional channel. Fig. 1 shows the shape of the particle. Calculated results of particle trajectories are shown in Fig. 2, where the scale of the longitudinal distance is greatly reduced so that the motion of the particles in the whole channel can be

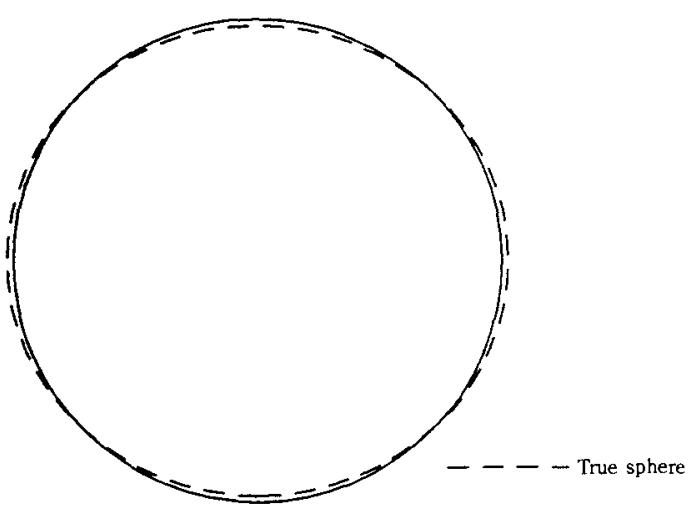

Fig. 1 Non-spherical particle ..... True sphere 
$500 \mathrm{~h}$

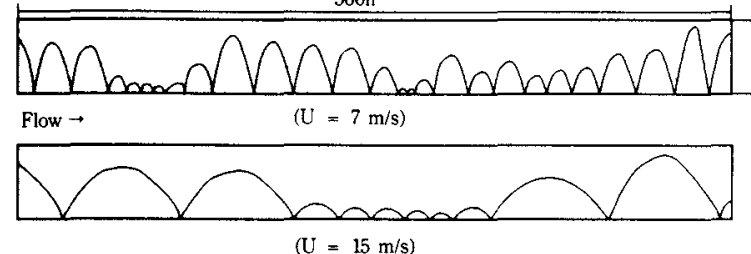

Fig. 2 Trajectories of a particle in a horizontal channel Particle size $=1.1 \mathrm{~mm}$, Particle density $=1038 \mathrm{~kg} / \mathrm{m}^{3}$ Restitution coefficient $\mathrm{e}=0.93$,

Friction coefficient $\mu_{\mathrm{f}}=0.28$

seen. It was found that even a small deviation from the sphere leads to a considerable irregular bouncing motion.

\subsection{Inter-particle collision}

As the particle concentration becomes higher, interparticle collision occurs more often and the loss of kinetic energy of the particles due to this collision cannot be neglected. Fortunately, as long as the particulate phase is dispersed, it is sufficient to consider only simple collision and not multiple collision. As in the particle-to-wall collision, the impulsive equations are solved to obtain the translation and angular velocities after collision assuming that the coefficients of restitution e and friction $\mu_{\mathrm{f}}$ are known (Tanaka et al., 1991).

\section{DSMC (direct simulation monte carlo) method}

The trajectories of individual particles are obtained by numerically integrating the equations of particle motion with a time interval $\Delta$ t. This integration process is straightforward. As described in the above section 3.2, the particle velocities after binary collision are calculated from the values before collision and the physical properties such as the coefficients of restitution and friction. However it is not easy to find pairs of particles colliding with each other when a large number of particles exist in the flow field. If we attempt to find such pairs from the trajectories of individual particles, the computation time would become extremely long as the number of particles increases. The DSMC method, proposed by Bird (1976) to solve the Boltzmann equation of rarefied gas flow has been developed to resolve this difficulty.

In dispersed gas-solid flow involving large particles, the particle inertia forces are dominant, and collisions against a particle or wall are assumed to occur instantaneously. Therefore the motion of the solid phase is similar to that of molecules in rarefied gas except that the kinetic energy of the particle fluctuat- ing motion tends to decay due to the inelastic and frictional characteristics of collision.

The DSMC method has already been applied to calculate particle motion in gas-solid flows by Kitron et al. (1990), Tanaka et al. (1991) and Tanaka et al. (1993). Tanaka et al. (1991) applied the DSMC method to fully developed flows in a vertical pipe and obtained results which agree satisfactorily with whose predicted by the deterministic method (Tanaka \& Tsuji, 1991). The outline of the DSMC method is as follows.

(i) Each simulated particle represents a large number of "physical" particles,

(ii) The time internal for numerical integration is taken sufficiently small compared with the mean free time of the particles, and thus inter-particle collisions are uncoupled from free particle motion.

(iii) The flow field is divided into small cells over which change in flow properties is small. Particles are allowed to collide through a Monte Carlo procedure.

(iv) The calculation of particle motion is carried out by repeating the following procedure:

First, the motions of all simulated particles in the time interval $\Delta t$ are calculated using the equations of motion without regard for interparticle collisions. If the calculated path of a particle crosses a solid wall, the velocity is replaced with the post-rebound velocity. Secondly, inter-particle collision during the time interval $\Delta t$ is searched by means of the Monte Carlo procedure. The collision counterpart and geometry of collision are also chosen with the Monte Carlo method. If a particle collides with another particle, the post-collision velocities of the colliding pair are calculated using the impulsive equations as described in Section 3.2. The particle velocities are replaced by the post-collision velocities, but without changing their positions.

Several schemes to find out whether a particle collides with another particle within a time interval have been proposed. We used the modified Nanbu method (Illner and Neunzert, 1987). The probability of inter-particle collision for a particular particle is evaluated from the particle properties included in the cell. The collision probability $\mathrm{P}_{\mathrm{i}}$ of particle $\mathrm{i}$ during a time interval $\Delta t$ is given by,

$P_{i}=\sum_{j=1}^{N} P_{i j}$,

where $\mathrm{N}$ is the number of simulated particles in the cell and $\mathrm{p}_{\mathrm{ij}}$, the probability of collision between the particle $\mathrm{i}$ and particle $\mathrm{j}$ in the cell during the time interval $\Delta \mathrm{t}$. 
WIn evaluating $\mathrm{p}_{\mathrm{ij}}$, it must be noted that each simulated particle represents many physical particles. $p_{i j}$ is not a collision probability between a single particle $\mathrm{i}$ and a single particle $\mathrm{j}$ but that for a physical particle in a field over which physical particles represented by simulated particles $\mathrm{i}$ and $\mathrm{j}$ are distributed homogeneously. Assuming the particles are spheres having a uniform diameter $d_{s}, p_{i j}$ is given by,

$\mathrm{p}_{\mathrm{ij}}=\frac{\mathrm{n}}{\mathrm{N}} \mathrm{d}_{\mathrm{s}}^{2} \mathrm{~g}_{\mathrm{ij}} \Delta \mathrm{t}$

Where $\mathrm{n}$ is the number density of the real flow at the corresponding position, and $\mathrm{g}_{\mathrm{ij}}$ is the magnitude of relative velocity between both particles.

According to the modified Nanbu method, the occurrence of inter-particle collision and the collision counterpart are decided using a random number $\mathrm{R}_{\mathrm{ND}}$ obtained from a uniform distribution which ranges from zero to unity. A "candidate" collision counterpart $\mathrm{j}$ is selected first using the following equation.

$\mathrm{k}=\left[\left[\mathrm{R}_{\mathrm{ND}} \times \mathrm{N}\right]\right]+1$,

where $\left[\left[\mathrm{R}_{\mathrm{ND}} \times \mathrm{N}\right]\right]$ is defined as the integer part of $R_{N D} \times N$. Particle $i$ is then assumed to collide with particle $\mathrm{k}$ during the previous time interval if:

$\mathrm{R}_{\mathrm{ND}}>\frac{\mathrm{k}}{\mathrm{N}}-\mathrm{p}_{\mathrm{ik}}$.

In this procedure, $p_{i j}$ for any particle combination in a cell must not exceed $1 / \mathrm{N}$. This condition is satisfied by selecting an appropriate time interval, since $\mathrm{p}_{\mathrm{ij}}$ is proportional to $\Delta \mathrm{t}$ as shown in $\mathrm{Eq}$. (2).

If the particle $\mathrm{i}$ collides with the particle $\mathrm{k}$, the velocity of particle $i$ (though not that of $k$ ) is replaced by the velocity after the collision. The relative position of the collision is also given using random numbers.

\section{Contact forces}

\subsection{Cundall \& Strack model}

As the particle concentration increases, almost all the particles are in contact with neighboring ones. Since each particle has elasticity, a particle assembly forms a complicated vibration system having multiple degrees of freedom. Moreover the contact points vary with time. The influence of a particle on other particles far from it propagates in a disturbance wave. It is very difficult to consider the interaction between one particle and remote ones. If the time interval in numerical calculation is chosen sufficiently small, it can be assumed that during a single time interval disturbances do not propagate from any particle further than its immediate neighbors. In other words, the instantaneous motion of each particle is determined by the contact forces acting between that particle and particles with which it is in contact. This assumption, proposed by Cundall and Strack (1979), makes it possible to save memory space. Based on this assumption, the motion of each particle in dense phase flows can be obtained by integrating the equations of motion step by step in which the contact forces between a particle and its immediate neighbors are taken into account. However, unlike dispersed phase flows, a method such as DSMC can not be used and trajectory calculation must be performed for all the physical particles.

In this simulation, the contact forces are more important then the inter-particle collision. Cundall and Strack (1979) proposed the model shown in Fig. 3 to formulate the contact forces between

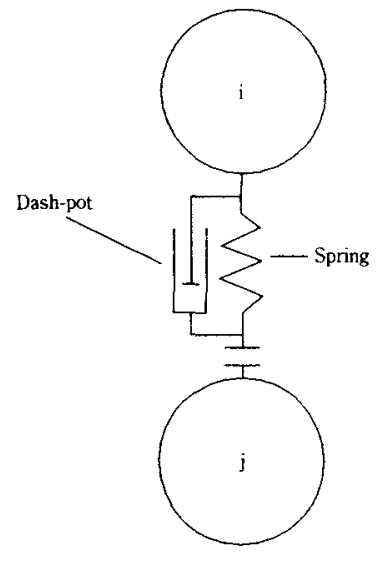

(a) Normal force

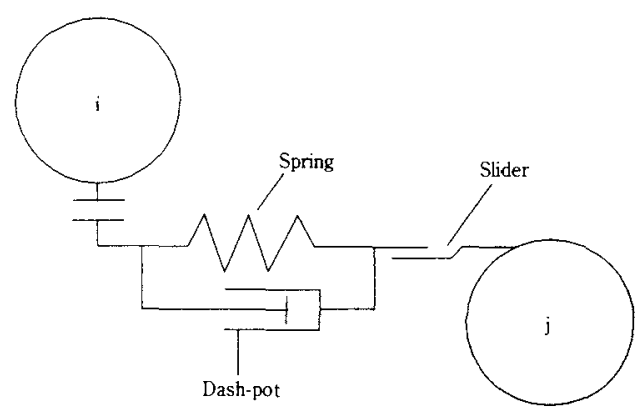

(b) Tangential force

Fig. 3 Model of contact forces 
two spheres. The model consists of a spring, a dashpot and a slider. The model in Fig. $\mathbf{3}$ is similar to the Voigt model known in the field of rheology. The effects of these mechanical elements on particle motion appear through the following parameters;

stiffness

damping coefficient

friction coefficient

When two particles denoted by $\mathrm{i}$ and $\mathrm{j}$ are in contact, the normal component of the contact force, $\mathbf{f}_{\text {Cnij }}$, acting on the Particle $\mathrm{i}$ is given by the sum of the forces due to the spring and the dash-pot;

$\overrightarrow{\mathrm{f}}_{\text {Cnij }}=\left(-\mathrm{k}_{\mathrm{n}} \delta_{\mathrm{nij}}-\eta_{\mathrm{n}} \overrightarrow{\mathrm{v}}_{\mathrm{rij}} \cdot \overrightarrow{\mathrm{n}}_{\mathrm{ij}}\right) \overrightarrow{\mathrm{n}}_{\mathrm{ij}}$

where

$\delta_{\text {nij }}:$ displacement of particle caused by the normal force

$\overrightarrow{\mathrm{v}}_{\mathrm{rij}}$ : velocity vector of particle i relative to particle j

$\overrightarrow{n_{i j}}$ : unit vector drawn from the center of particle $i$ to that of particle $j$

The tangential component of the contact force, $\vec{f}_{\mathrm{Ctij}}$, is given by

$\overrightarrow{\mathrm{f}}_{\mathrm{Ctij}}=-\mathrm{k}_{\mathrm{t}} \vec{\delta}_{\mathrm{tij}}-\eta_{\mathrm{tj}} \overrightarrow{\mathrm{v}}_{\mathrm{sij}}$

where $\mathrm{k}_{\mathrm{t}}$ and $\vec{\delta}_{\mathrm{tij}}$ are, respectively, the stiffness and displacement in the tangential direction. In the above equations, the suffixes $n$ and $t$ represent the components corresponding to the normal and tangential directions, respectively. $\vec{v}_{\mathrm{sij}}$ is the slip velocity of the contact point, which is given by

$$
\begin{aligned}
\vec{v}_{\text {sij }} & =\vec{v}_{r i j}\left(\vec{v}_{r i j} \cdot \vec{n}\right) \vec{n} \\
& +r_{s}\left(\vec{\omega}_{i}+\vec{\omega}_{j}\right) \times \vec{n}
\end{aligned}
$$

where $r_{s}$ is the radius of the sphere. If the following relation is satisfied

$\left|\mathrm{f}_{\text {Ctij }}\right|>\mu_{\mathrm{f}}\left|\overrightarrow{\mathrm{f}}_{\mathrm{Cnij}}\right|$

particle $\mathrm{i}$ slides and the tangential force is given by

$$
\overrightarrow{\mathrm{f}}_{C_{\text {tij }}}=-\mu_{\mathrm{f}}\left|\overrightarrow{\mathrm{f}}_{\mathrm{Cnij}}\right| \overrightarrow{\mathrm{t}}_{\mathrm{ij}}
$$

instead of Eq. (6). Eq. (9) is the Coulomb-type friction law. $\overrightarrow{\mathrm{t}}_{\mathrm{ij}}$ in $\mathrm{Eq} .(9)$ is the unit vector defined by

$$
\overrightarrow{\mathrm{t}}_{\mathrm{ij}}=\overrightarrow{\mathrm{v}}_{\mathrm{sij}} /\left|\overrightarrow{\mathrm{v}}_{\mathrm{sij}}\right|
$$

The same relations as the above equations are derived for the contact with the wall if particle $j$ is replaced by the wall.

In general, several particles are in contact with particle $i$ at the same time. Therefore the total force and torque acting on particle $i$ is obtained by taking the sum of the above forces with respect to $\mathrm{j}$.

\subsection{Determination of parameters in Cundall \& Strack model}

The next step after modeling the contact forces is to determine the values of the stiffness $\mathrm{k}$, damping coefficient $\eta$ and friction coefficient $\mu_{\mathrm{f}}$. Among these parameters, the friction coefficient $\mu_{\mathrm{f}}$ is measurable and regarded as a parameter given empirically.

Fortunately the stiffness can be calculated using the Hertzian contact theory when the physical properties such as the Young's modulus and Poisson ratio are known. According to the Hertizian contact theory, the relation between the normal force $\mathrm{P}_{\mathrm{n}}$ and displacement $\delta_{\mathrm{n}}$ is given by

$\mathrm{P}_{\mathrm{n}}=\mathrm{K}_{\mathrm{n}} \delta_{\mathrm{n}}^{3 / 2}$

$\mathrm{K}_{\mathrm{n}}$ is given as a function of the physical properties of two particles. If the two particles have the same properties, $\mathrm{K}_{\mathrm{n}}$ is expressed as

$K_{n}=\frac{\sqrt{2 r_{s}} E_{s}}{3\left(1-\sigma_{s}^{2}\right)}$

where $r_{s}:$ radius

$\mathrm{E}_{\mathrm{s}}$ : Young's modulus of the particle

$\sigma_{\mathrm{s}}:$ Poisson ratio of the particle

The above equation signifies that the force varies with the $3 / 2$ power of the displacement. Therefore if the above results are applied to the model of the contact forces given in the foregoing section, Eq. (5) can be replaced by

$\overrightarrow{\mathrm{f}}_{\mathrm{Cnij}}=\left(-\mathrm{K}_{\mathrm{n}} \delta_{\mathrm{nij}} 3 / 2-\eta_{\mathrm{nj}} \overrightarrow{\mathrm{v}}_{\mathrm{rij}} \cdot \overrightarrow{\mathrm{n}}_{\mathrm{jij}}\right) \overrightarrow{\mathrm{n}}_{\mathrm{ij}}$

As for the relation between the tangential force $\mathrm{P}_{t}$ and displacement, theories developed by Mindlin (1949) and Mindlin and Deresiewicz (1953) are used (Tsuji et al., 1992). 
Regarding the damping coefficient, Cundall and Strack (1979) proposed two expressions, given by the following equations,

$\eta_{\mathrm{n}}=2{\sqrt{\mathrm{m} / \mathrm{k}_{\mathrm{n}}}}$

$\eta_{\mathrm{t}}=2 \sqrt{\mathrm{m} / \mathrm{k}_{\mathrm{t}}}$

which are derived from the condition of the critical damping of a single degree-of-freedom system consisting of a mass, spring and dash-pot.

We (Tsuji et al., 1992) proposed another method for determining the damping coefficient. In our method, the damping coefficient is related to the coefficient of restitution which is regarded as one of particle's physical properties or can be measured in a simple experiment.

\section{Equation of particle motion}

Individual particles have two types of motion; translational and rotational motions. The translational motion is caused by the contact force, fluid force and gravitational force. The rotational motion in dispersed phase flows is caused by collision with the wall and particles. The rotational motion in the fluid is affected by viscous dissipation. In dense phase flows where particles are in contact with neighboring ones, the rotational motion is caused by the tangential components of the contact forces.

The equations of translational and rotational motions are

$$
\begin{aligned}
& \ddot{\vec{r}}=\left(\overrightarrow{\mathrm{f}}_{\mathrm{c}}+\overrightarrow{\mathrm{f}}_{\mathrm{D}}\right) / \mathrm{m}+\overrightarrow{\mathrm{g}} \\
& \dot{\vec{\omega}}=\overrightarrow{\mathrm{T}}_{\mathrm{c}} / \mathrm{I}
\end{aligned}
$$

where $\vec{r}$ : position vector of the particle's gravity center

m : particle mass

$\overrightarrow{f_{c}}$ : sum of contact forces

$\overrightarrow{\mathrm{f}_{\mathrm{D}}}$ : sum of fluid forces

$\overrightarrow{\mathrm{g}}$ : gravity acceleration vector

$\overrightarrow{\mathrm{T}}_{\mathrm{c}}$ : sum of torque

I. : moment of inertia of the particle

( ) : time derivative

The new velocity and position after a time interval $\Delta t$ are expressed as:

$$
\overrightarrow{\mathrm{v}}_{\mathrm{s}}=\overrightarrow{\mathrm{v}}_{\mathrm{s} 0}+\overrightarrow{\mathrm{r}}_{0} \Delta \mathrm{t}
$$

$$
\begin{aligned}
& \overrightarrow{\mathrm{r}}=\overrightarrow{\mathrm{r}}_{0}+\overrightarrow{\mathrm{v}}_{\mathrm{s}} \Delta \mathrm{t} \\
& \vec{\omega}=\vec{\omega}_{0}+\vec{\omega}_{0} \Delta \mathrm{t}
\end{aligned}
$$

where $\vec{v}$ : velocity vector

suffix 0 : previous value

suffix s: particle

Regarding the fluid forces, drag, lift (due to rotation and velocity shear) and torque are considered. Various theoretical or empirical formulae are available for such forces acting on particles freely suspended in the fluid. In dense phase flows, the lift and torque due to viscosity can be neglected, and only the drag is taken into account. Such drag can be estimated by considering the pressure gradient caused by the fluid passing through the particle bed, as might be found from established empirical formulae such as the Ergun equation (Ergun, 1952).

\section{Equation of fluid motion}

It is almost impossible even for modern supercomputers to solve the problem of the instantaneous flow field of small scale between moving particles in multiphase flows. Furthermore, the scale of the phenomena of interest to us is much larger than the size of individual particles. Therefore it is reasonable to perform calculations based on locally averaged quantities according to Anderson and Jackson (1967). As is usual in many numerical calculations of flow fields, the finite difference method can be used to obtain the flow field in the simulation of multiphase flows. Therefore, the flow domain is divided into cells, the size of which is smaller than the macroscopic motion but larger than the particle size. All the quantities such as pressure $\mathrm{p}$ and velocity $\mathrm{u}$ are averaged in the cell using a weight function. The void fraction $\varepsilon$ of each cell can be defined by the number of particles existing in the cell.

The equation of continuity is given by

$\frac{\partial \varepsilon}{\partial \mathrm{t}}+\frac{\partial}{\partial \mathrm{x}_{\mathrm{i}}}\left(\varepsilon \mathrm{u}_{\mathrm{j}}\right)=0$

The equation of fluid motion is

$\frac{\partial}{\partial x_{i}}\left(\varepsilon u_{i} u_{j}\right)=-\frac{\varepsilon}{p} \frac{\partial p}{\partial x_{i}}+f_{s i}$

where $p$ is the fluid density. The last term in Eq. (21) denotes the mutual interaction between particles 
and fluid. Eq. (21) signifies that the fluid is assumed to be inviscid. The interaction term depends on the fluid properties, the void fraction and the relative velocity between particles. For details, refer to Tsuji et al., (1993).

\section{Calculation procedure}

As an example of the calculation procedure, flow charts in the simulation of a fluidized bed are given in Fig. 4.

\section{Examples of simulation}

\subsection{Without fluid}

Our group in Osaka University has developed computer programs for fluidized beds and pneumatic conveying, in which the fluid forces play important roles. However, simulations neglecting fluid flow also have many applications in powder technology. In fact we have attempted to predict various particulate flows such as particle discharge from hoppers and particle mixing in rotating vessels. In this section, such examples are shown first.

Fig. 5 illustrates the mixing of two types of particles in a rotating square duct. This simulation was made three-dimensionally. From this simulation, the time required for mixing and the optimal rotating speed can be predicated.

Fig. 6 illustrates a particle flow from a round hopper. Initially, two types of particles are uniformly mixed in the upper hopper. The dark color particles are heavy particles and the light color particles are light ones. As the particles are discharged, particle segregation is observed, that is, the heavy particles tend to occupy the lower and center parts of the lower hopper.

Fig. 7 also illustrates particle flows from round hoppers. The effect of a cone near the gate on the flow pattern can be clearly observed. The case without the cone shows a funnel-type flow pattern while the case with the cone shows a mass flow.

\subsection{With fluid}

Fig. 8 (Tanaka et al., 1993) illustrates a dispersed gas-solid flow in a vertical channel, where the DSMC method was used. Note that the vertical scale of the channel is compressed in these figures. When the superficial gas velocity is large or the mean solid mass flux is small, the particle concentration has a nearly homogeneous distribution. As the gas velocity decreases and the solid loading increases,

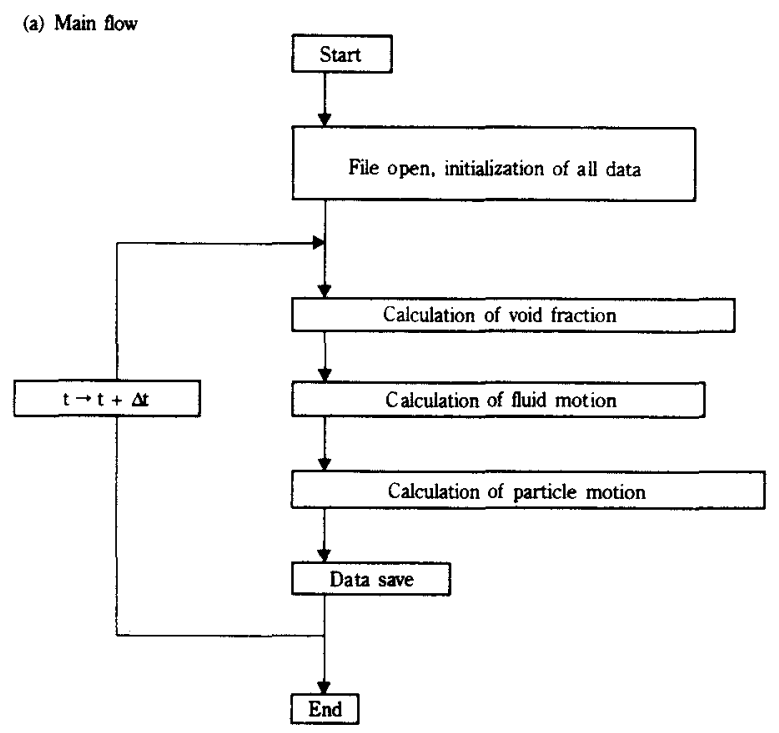

Fig. 4 (a) Flow chart

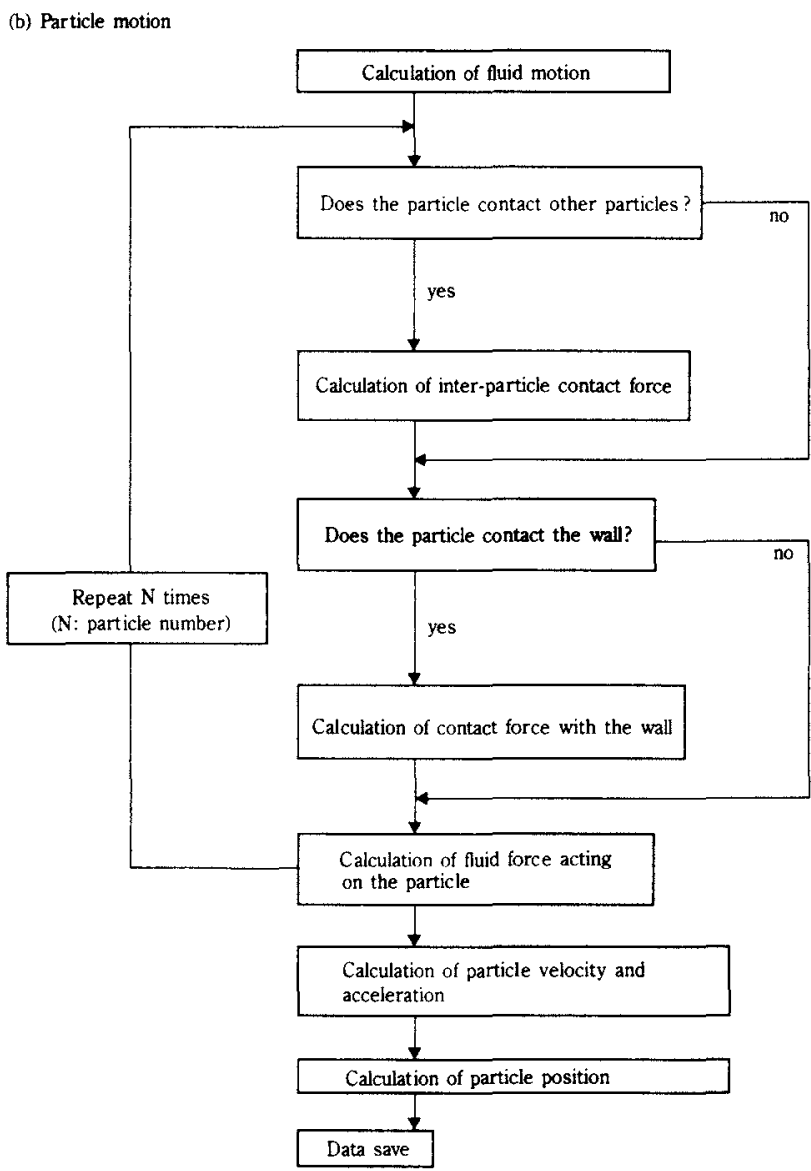

Fig. 4 (b) Flow chart

particle clusters in which the solid volume fraction is extremely large are formed near the wall. These particle clusters substantially affect the gas flow. The gas velocity and particle velocity are reduced in particle clusters and in their wakes. The velocities 

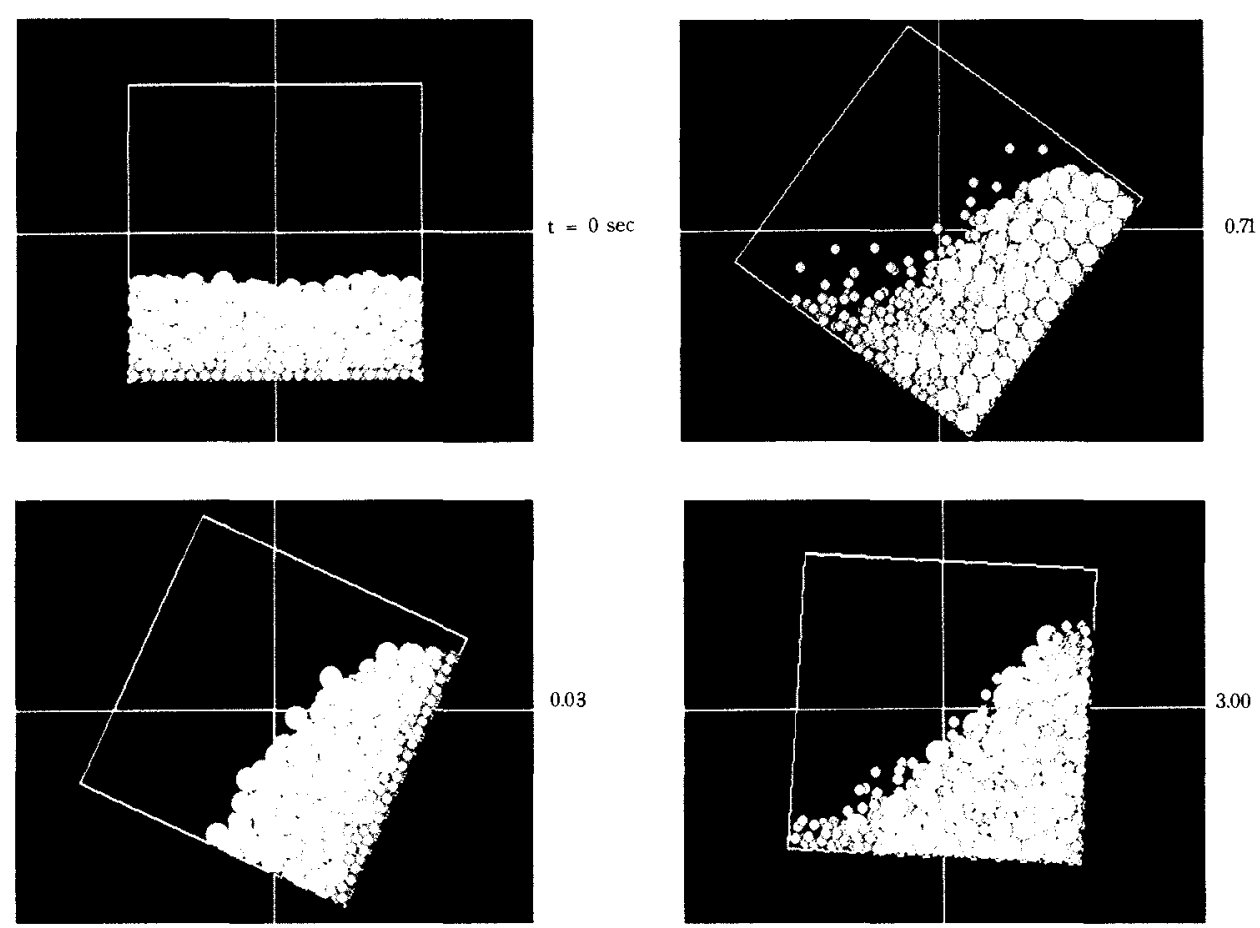

Fig. 5 Particle mixing in a rotating duct

Side length of the dust $=50 \mathrm{~mm}$,

Depth of the dust $=25 \mathrm{~mm}$

Rotation speed $=120 \mathrm{RPM}$

Properties of large particles:

Size $=4 \mathrm{~mm}$, Number $=300$, Density $=2700 \mathrm{~kg} / \mathrm{m}^{3}$

Properties of small particles:

Size $=2 \mathrm{~mm}$, Number $=200$, Density $=2700 \mathrm{~kg} / \mathrm{m}^{3}$
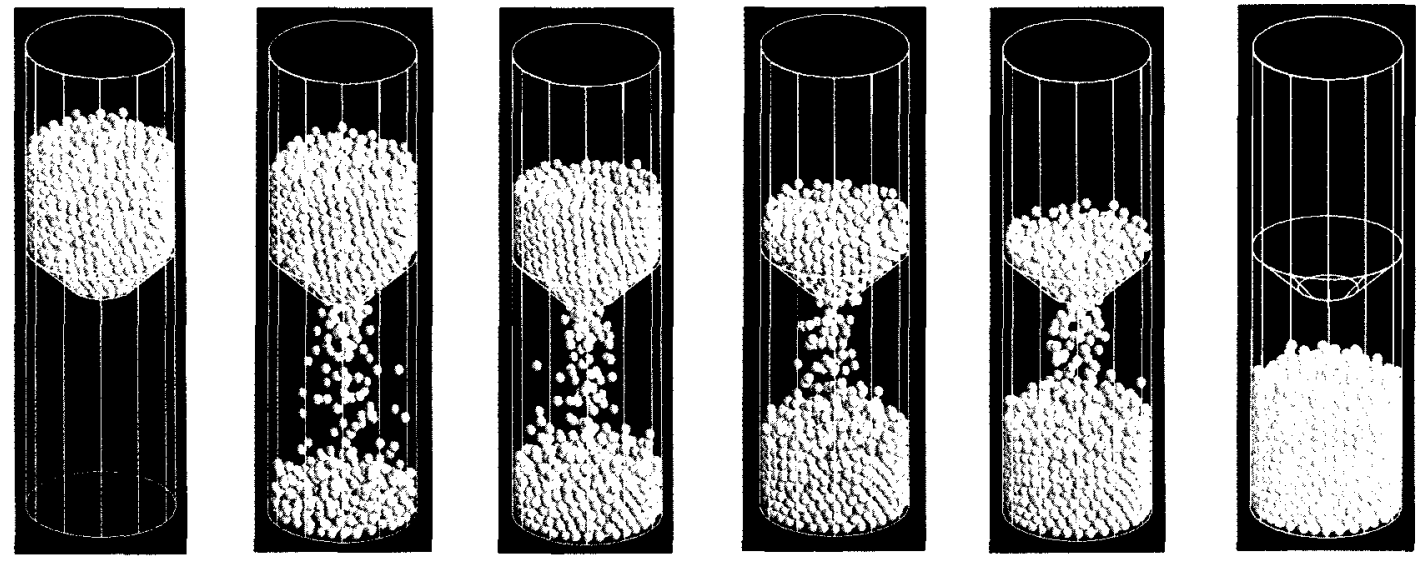

Fig. 6 Particles discharging from a cylindrical hopper

Internal diameter of the hopper $=50 \mathrm{~mm}$

Diameter of the hole $=20 \mathrm{~mm}$

Properties of heavy particles:

Size $=4 \mathrm{~mm}$, Number $=950$, Density $=2700 \mathrm{~kg} / \mathrm{m}^{3}$

Properties of light particles:

Size $=4 \mathrm{~mm}$, Number $=950$, Density $=1000 \mathrm{~kg} / \mathrm{m}^{3}$

of particles in dense clusters can even become negative, meaning that they drop down along the wall.

Fig. 9 represents a flow pattern obtained in a channel wider than that of Fig. 8, giving rise to clusters forming not only along the walls but also in the central part of the channel. The close-up of a typical V-shaped cluster formed in the central part of the channel is also shown in Fig. 10. The scale of the close-up is not compressed.

Fig. 11 (Tsuji et al., 1992) illustrates a plug flow 

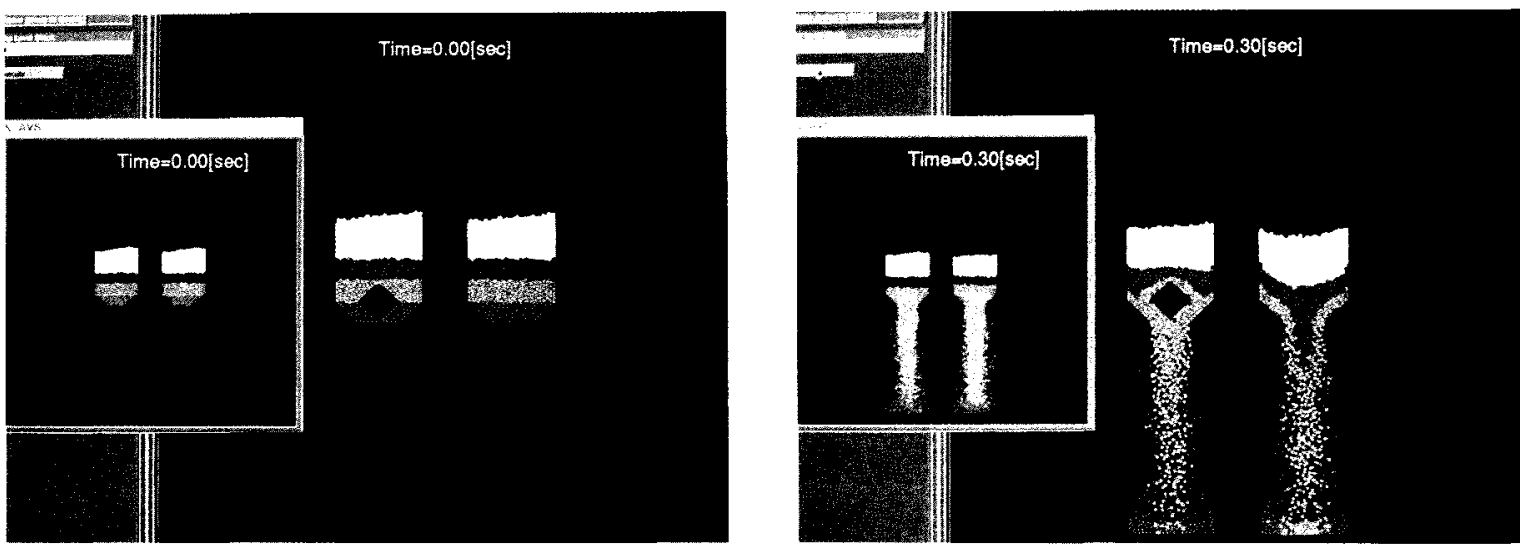

Fig. 7 Particles discharging from a cylindrical hopper (Comparison between two cases with and without an inserted cone, Pictures in the large frame show the view in the vertical central plane inside the hopper. Pictures in the small frame show side views.)

Internal diameter of the hopper $=80 \mathrm{~mm}$ Diameter of the hole $=40 \mathrm{~mm}$

Properties of particles: Size $=4 \mathrm{~mm}$, Number $=10,000$, Density $=3000 \mathrm{~kg} / \mathrm{m}^{3}$

Fig. 7 (a) Before discharge

Fig. 7 (b) Without a cone, the flow at the outlet is a funnel-type flow. The cone is effective for producing a mass flow pattern.

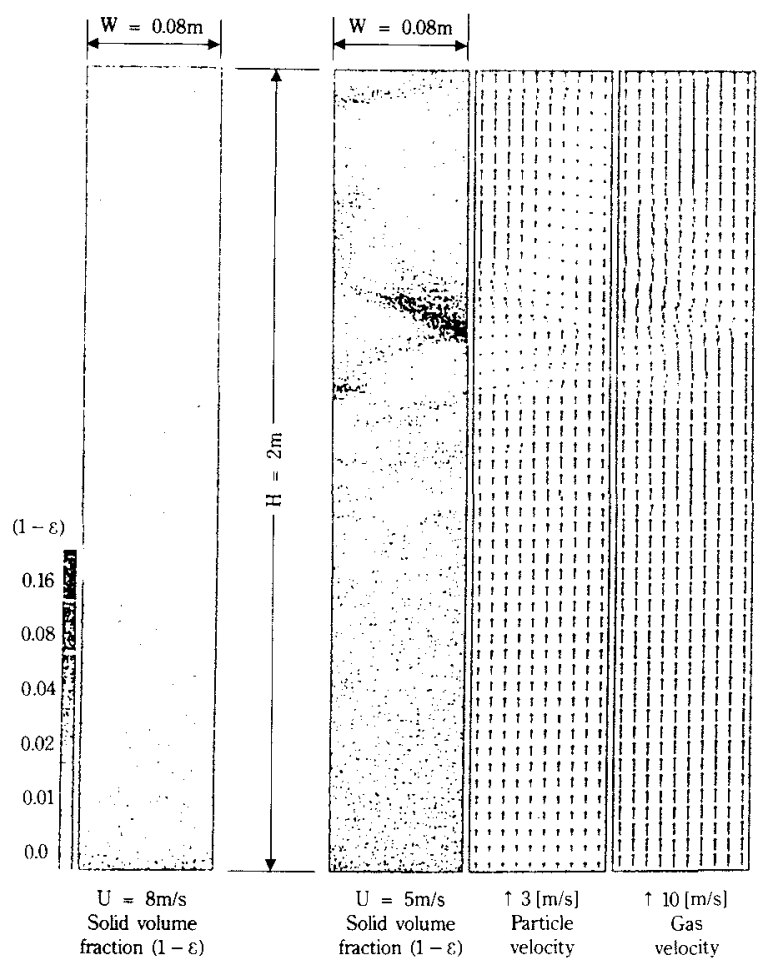

Fig. 8 Flow pattern of dispersed gas-solid flow in a vertical channel

Channel width $\mathrm{W}=0.08 \mathrm{~m}$

Particle mass flux $Q=25 \mathrm{~kg} / \mathrm{m}^{2} \mathrm{~s}$,

Restitution coefficient $\mathrm{e}=0.94$,

Friction coefficient $\mu_{\mathrm{f}}=0.28$

of cohesionless particles in a horizontal pipe. It can clearly be observed that the plug gives rise to a wave-like motion similar to that of a gas-liquid slug flow. There are stationary particles between the

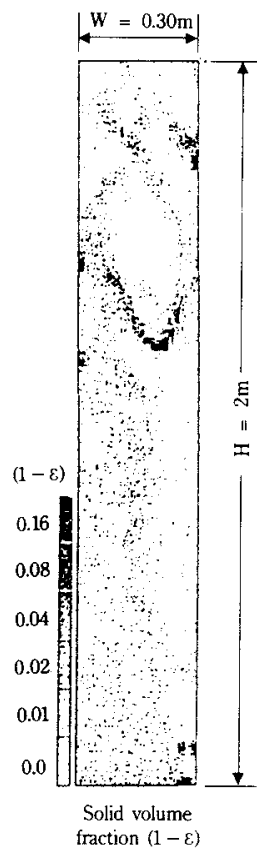

Fig. 9 Flow pattern of dispersed gas-solid flow in a vertical channel

$\left(W=0.30 \mathrm{~m}, \mathrm{Q}=25 \mathrm{~kg} / \mathrm{m}^{2} \mathrm{~s}, \mathrm{e}=0.94, \mathrm{u}_{\mathrm{f}}=0.28\right)$

plugs. The plug sweeps up the stationary particles in front and leaves behind a stationary layer. These calculated results agree well with the actual phenomena observed in experiments. 


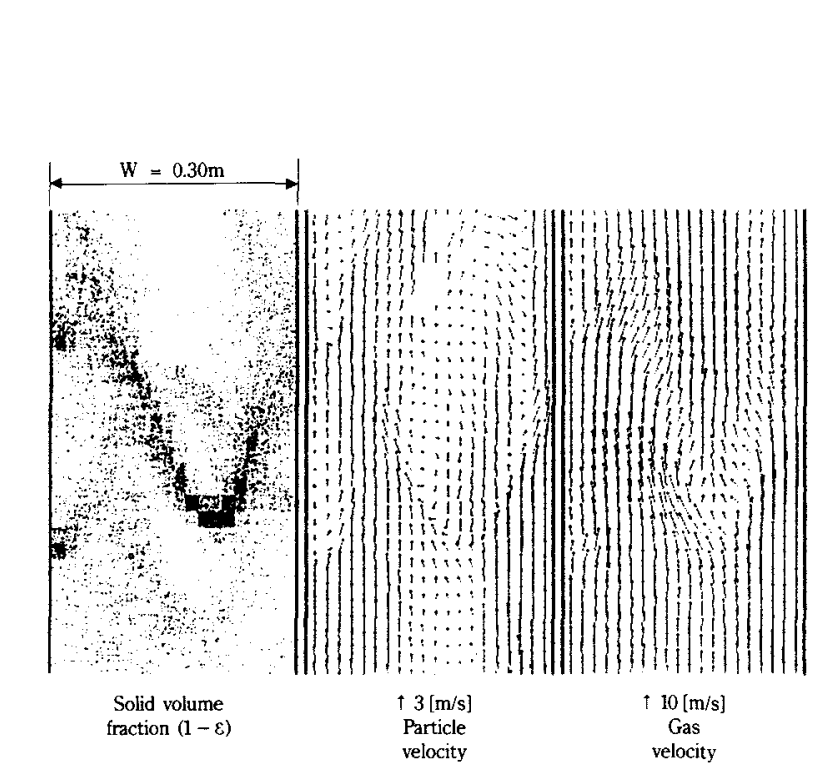

Fig. 10 Flow pattern of dispersed gas-solid flow in a vertical channel.

$\left(W=0.30 \mathrm{~m}, \mathrm{Q}=25 \mathrm{~kg} / \mathrm{m}^{2} \mathrm{~s}, \mathrm{e}=0.94, \mathrm{u}_{\mathrm{f}}=0.28\right)$

We have made simulations of fluidized beds having various geometries. Although the simulation is based on simple models, the formation of bubbles and slugs is observed in the same way as in physical experiments. Fig. 12 represents an example of such simulations. In this case, the particle motion was treated two-dimensionally. The air is issued from three nozzles provided at the bottom.

\section{Concluding remarks}

The author has not reviewed sufficiently the studies made by other researchers in this review. It should be noted that discrete particle simulations without fluids have been made actively in the field of soil and rock mechanics.

It has not been long since discrete particle simulation was applied in powder technology. As computer
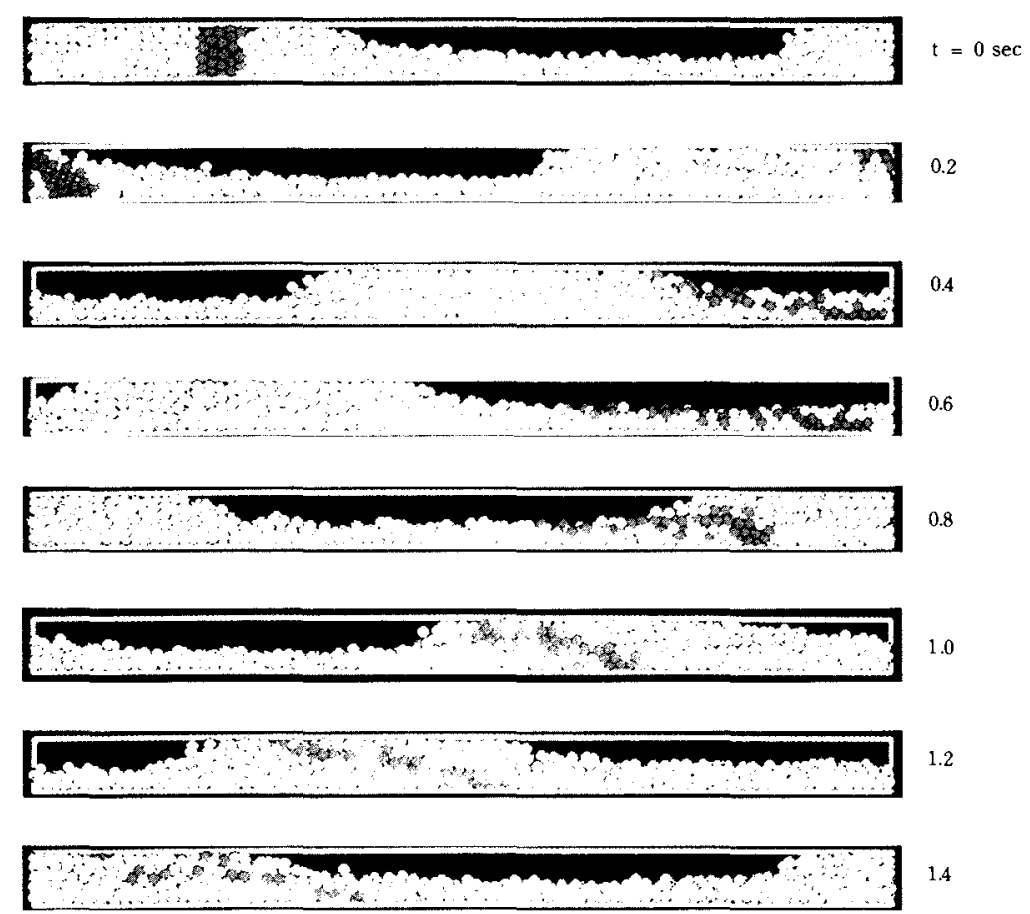

Fig. 11 Dense phase pneumatic conveying in a horizontal pipe

Pipe internal diameter $=50 \mathrm{~mm}$ Superficial air velocity $=2.4 \mathrm{~m} / \mathrm{s}$

Properties of particles: Size $=10 \mathrm{~mm}$, Number $=1000$, Density $=1000 \mathrm{~kg} / \mathrm{m}^{3}$
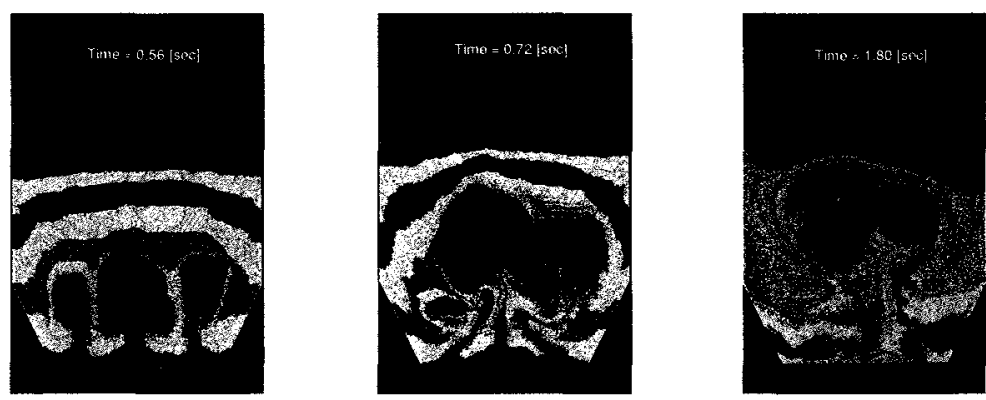

Fig. 12 Two-dimensional fluidized bed with three jets. Number of particles $=30,000$ 
power increases, this method will be all the more attractive and useful. As far as particle motion is concerned, we have already succeeded in obtaining qualitatively good correlations. It is expected that from now, research in discrete particle simulation will be developed along the two directions; seeking quantitative correlations or including various influential factors such as effects of humidity, cohesion forces, particle shape and so on. Recently, a commercial software "P-TAK" based on part of our program has been developed for the prediction of particle flows in hoppers and fluidized beds by Yabushita et al., (1993).

\section{Nomenclature}

D : pipe diameter

$\mathrm{d}_{\mathrm{s}} \quad$ : particle diameter

$\mathrm{E}_{\mathrm{s}} \quad$ : Young's modulus

e : coefficient of restitution

$\overrightarrow{\mathrm{f}_{\mathrm{C}}} \quad:$ contact force

$\overrightarrow{\mathrm{f}_{\mathrm{D}}} \quad$ : fluid drag force

$\mathrm{G}_{\mathrm{s}} \quad$ : shear modulus

$\overrightarrow{\mathrm{g}} \quad$ : gravity acceleration vector

I : moment of inertia of particle

$\mathrm{k} \quad$ : stiffness

m : particle mass

$\mathrm{N} \quad$ : number of particles

$\overrightarrow{\mathrm{n}} \quad$ : unit vector in the normal direction

$\mathrm{P} \quad$ : elastic force

p : pressure

Q : particle mass flux

Re : Reynolds number

$\vec{r} \quad$ : position vector of particle's gravity center

$r_{\mathrm{s}}:$ particle radius

$\mathrm{t}:$ : time

$\overrightarrow{\mathrm{t}} \quad$ : unit vector in the tangential direction

$\overrightarrow{\mathrm{T}}_{\mathrm{C}} \quad$ : torque

$\mathrm{U}$ : bulk gas velocity

u : gas velocity

$\overrightarrow{\mathrm{V}}_{\mathrm{s}} \quad$ : particle velocity vector

$\delta \quad:$ displacement

$\varepsilon \quad$ : void fraction (porosity)

$\eta \quad$ : damping coefficient

$\mu_{\mathrm{f}} \quad:$ friction coefficient

$p \quad:$ air density

$\sigma \quad$ : Poisson ratio

$\vec{\omega} \quad$ : angular velocity vector of particle

suffix

n : normal component

$\mathrm{t}:$ : tangential component

\section{References}

1) Anderson, T. B and Jackson, R., "A Fluid Mechanical Description of Fluidized Beds", I \& EC Fundamentals, 6, 527. [1967]

2) Bird, G. A., "'Molecular Gas Dynamics”, Oxford Univ. Press, p. 118, [1976]

3) Cundall, P. A. and O. D. Strack, O. D., "A discrete numerical model for granular assemblies", Geotechnique 29-1, 47, [1979]

4) Ergun, S., "Fluid flow through packed bed", Chem. Eng. Progr., 48, 89, [1952]

5) Frank, T., Schade, K. P. and Petrak, D., "Numerical simulation and experimental investigation of a gas-solid two-phase flow in a horizontal channel', Int. J. Multiphase Flow, 19-1, 187, [1993]

6) Illner, R. and Neunzert, H., "On simulation methods for the Boltzmann equation", Transport Theory and Statistical Physics, 16, 141, [1987]

7) Kitron, A., Elperin, T., and Tamir, A., "Monte Carlo Simulation of Gas-Solids Suspension Flows in Impinging Streams Reactors", Int. J. Multiphase Flow, 16-1, 1, [1990]

8) Matsumoto, S. and Saito, S., "On the mechanism of suspensions in horizontal pneumatic conveying: Monte Carlo Simulation based on the irregular bouncing model', J. Chem. Engng Japan 3-1, 83, [1970a]

9) Matsumoto, S. and Saito, S., "Monte Carlo Simulation of horizontal pneumatic conveying based on the rough wall model' ', J. Chem. Engng Japan 3-2, 223, [1970b]

10) Mindlin, R.D., "Compliance of elastic bodies in contact", Appl. Mech. (Trans. ASME), 16, 259, [1949]

11) Mindlin, R. D. and Deresiewicz, H., "Elastic spheres in contact under varying oblique forces", J. Appl. Mech. (Trans. ASME). 20, 327 [1953]

12) Patankar, S. V., “Numerical Heat Transfer and Fluid Flow', Hemisphere Publishing Corporation, [1980]

13) Tanaka, T. and Tsuji, Y., "Numerica Simulation of Gas-Solid Two-Phase Flow in a Vertical Pipe: On the Effect of Inter Particle Collision", ASME/FED-Vol. 121, Gas-Solid Flows, 123. [1991]

14) Tanaka, T., Kiribayashi, K. and Tsuji, Y., "Monte Carlo Simulation of Gas-Solid Flow in Vertical Pipe or Channel", Proc. Int. Conf. on Multiphase Flows '91-Tsukuba, Tsukuba Japan, 
Vol. 2, 439, [1991]

15) Tanaka, T., Yonemura, S. and Tsuji, Y., " "Cluster Formation in Gas-Solid Flow Predicted by DSMC Method", 5th Int, Symp. on Gas-Solid Flows, ASME Fluids Engineering Conference, Washington DC, [1993]

16) Tsuji, Y. et al., "Numerical simulation of gassolid two-phase flow in a two-dimensional horizontal channel", Int. J. Multiphase Flow, 13-5, 671, [1987]

17) Tsuji, Y., Shen, N-Y. and Morikawa, Y., "Numerical simulation of gas-solid flows. I (Particleto-wall collision)", Techn. Rep. Osaka University, 39-1975, 233, [1989]
18) Tsuji, Y., Tanaka, T. and Ishida, T., "Lagrangian numerical simulation of plug flow of cohesionless particles in a horizontal pipe", Powder Technology, 71, 239, [1992]

19) Tsuji, Y., Kawaguchi, T. and Tanaka, T., "Discrete Particle Simulation of Two-Dimensional Fluidized Bed", Powder Technology, 77, 79, [1993]

20) Yabushita, Y., Shibata, K., Saido, K. and Tsuji, Y., "Development of a Two-Dimensional Transient Code for Simulation Behavior in Hoppers and Fluidized Beds --- Models and Code Verifications", 2nd Int. Conf. on Micromechanics of Granular Media (Powders and Grains 93), [1993]

\section{Author's short biography}

\section{Yutaka Tsuji}

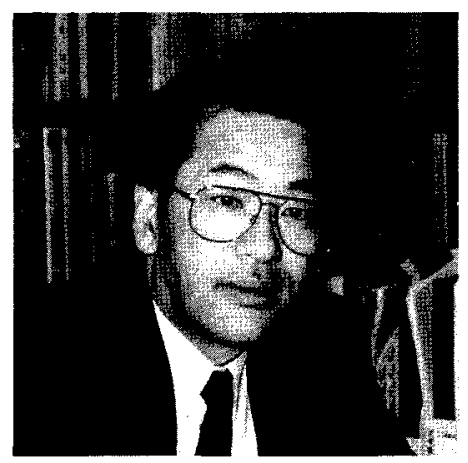

Prof. Yutaka Tsuji has been at the Osaka University, Japan since 1970. His major research interest lies in numerical simulation of multiphase flow and optical measurement of fluid-solid two-phase flow. He is the coordinator of the Simulation Committee of the Association of Powder Process Industry \& Engineering, Japan. 